\title{
Lusioersily
}

\section{Active Physical Practice Followed by Mental Practice Using BCl-Driven Hand Exoskeleton: A Pilot Trial for Clinical Effectiveness and Usability}

Chowdhury, A., Meena, Y. K., Haider, R., Bhushan, B., Uttam, A. K., Pandey, N., Hashmi, A. A., Bajpai, A., Dutta, A., \& Prasad, G. (2018). Active Physical Practice Followed by Mental Practice Using BCl-Driven Hand Exoskeleton: A Pilot Trial for Clinical Effectiveness and Usability. IEEE Journal of Biomedical and Health Informatics, 22(6), 1786-1795. https://doi.org/10.1109/JBHI.2018.2863212

Link to publication record in Ulster University Research Portal

Published in:

IEEE Journal of Biomedical and Health Informatics

Publication Status:

Published online: 03/08/2018

DOI:

10.1109/JBHI.2018.2863212

\section{Document Version}

Author Accepted version

\section{General rights}

Copyright for the publications made accessible via Ulster University's Research Portal is retained by the author(s) and / or other copyright owners and it is a condition of accessing these publications that users recognise and abide by the legal requirements associated with these rights.

\section{Take down policy}

The Research Portal is Ulster University's institutional repository that provides access to Ulster's research outputs. Every effort has been made to ensure that content in the Research Portal does not infringe any person's rights, or applicable UK laws. If you discover content in the Research Portal that you believe breaches copyright or violates any law, please contact pure-support@ulster.ac.uk. 


\title{
Active Physical Practice Followed by Mental Practice Using BCI-Driven Hand Exoskeleton: A Pilot Trial for Clinical Effectiveness and Usability
}

\author{
Anirban Chowdhury, Student Member, IEEE, Yogesh Kumar Meena, Member, IEEE, Haider Raza, Member, IEEE, \\ Braj Bhushan, Ashwani Kumar Uttam, Nirmal Pandey, Adnan Ariz Hashmi, Alok Bajpai, \\ Ashish Dutta, Member, IEEE, and Girijesh Prasad, Senior Member, IEEE
}

\begin{abstract}
Appropriately combining mental practice (MP) and physical practice (PP) in a post-stroke rehabilitation is critical for ensuring a substantially positive rehabilitation outcome. Here we present a rehabilitation protocol incorporating a separate active PP stage followed by MP stage, using a hand exoskeleton and brain-computer interface (BCI). The PP stage was mediated by a force sensor feedback based assist-as-needed control strategy, whereas the MP stage provided BCI based multimodal neurofeedback combining anthropomorphic visual feedback and proprioceptive feedback of the impaired hand extension attempt. A 6 week long clinical trial was conducted on 4 hemiparetic stroke patients (screened out of 16) with a left-hand disability. The primary outcome, motor functional recovery, was measured in terms of changes in Grip-Strength (GS) and Action Research Arm Test (ARAT) scores; whereas the secondary outcome, usability of the system was measured in terms of changes in mood, fatigue, and motivation on a visual-analog-scale (VAS). A positive rehabilitative outcome was found as the group mean changes from the baseline in the GS and ARAT were $+6.38 \mathrm{~kg}$ and +5.66 accordingly. The VAS scale measurements also showed betterment in mood $(-1.38)$, increased motivation $(+2.10)$ and reduced fatigue $(-0.98)$ as compared to the baseline. Thus the proposed neurorehabilitation protocol is found to be promising both in terms of clinical effectiveness and usability.
\end{abstract}

Index Terms-BCI, EEG, Exoskeleton, Neurofeedback, Neurorehabilitation, Stroke.

\section{INTRODUCTION}

G lobally over $80 \%$ of the stroke survivors suffer from some form of disability out of which $85 \%$ may have serious upper-limb movement deficits [1]. Although there remains a good chance of recovery in the first few months after stroke [2], after 6 months post-stroke $65 \%$ of them suffer from a permanent disability of the affected limb leading to degraded quality of life [3]. The clinical effectiveness of conventional therapies is limited by their passive nature, especially for the hand function, which is considered to be the most difficult problem for stroke rehabilitation [4]. Therefore new intervention techniques are being extensively explored.

A. Chowdhury and A. Dutta are with the Centre of Mechatronics, Indian Institute of Technology (IIT) Kanpur, Kanpur-208016, India

Y. K. Meena and G. Prasad are with Intelligent Systems Research Centre, Ulster University, Derry Londonderry-BT48 7JL, UK

H. Raza is with School of Computer Science and Electronic Engineering, University of Essex, Colchester-CO4 3SQ, UK

B. Bhushan is with the Department of Humanities and Social Sciences, Indian Institute of Technology (IIT) Kanpur, Kanpur-208016, India

A. K. Uttam, N. Pandey, A. A. Hashmi, and A. Bajpai are with Regency Hospital, Kanpur, Kanpur-208019, India
Since it has been found that the similar neuro-muscular structures are associated with the physical practice and the kinesthetic imagery of the same motor activity, brain-computer interface (BCI) has become an essential means for designing advanced neurorehabilitation techniques [5]. The transition from assistive to rehabilitative use of BCI requires special focus on the reinforcement of the brain-networks related with the long-term restoration of the lost motor functions [6], such as contralateral connectivity between the primary and somatosensory motor cortex [7]. In this regard the BCI based synchronous cortical and peripheral stimulation have been found to be very effective to enhance corticospinal excitability [8]. It facilitates the patient to directly observe the ongoing cortical activity in terms of contingent visual or orthotic feedback [9], [10], [11]. In a controlled study by Naros et al. [12] established the effectiveness of contingent neurofeedback. Indeed, the contingent proprioceptive feedback was found to be more effective than the visual feedback for motor skill learning [13].

Systematic controlled trials have shown that BCI along with robotic therapy yielded better performance than conventional robotic therapy in terms of motor recovery outcomes [14], [15], [16]. A BCI based training with discrete movement feedback of a virtual hand was also found to be feasible and tolerable for the stroke patients [17], [18]. A functional electrical stimulus (FES) has been used as an orthotic feedback, triggered by BCI, which has shown significant recovery in index finger extension [19]. A broad review of studies conducted on upper-limb rehabilitation for the last few decades has shown that mental practice (MP) in conjunction with the physical practice (PP) is an essential criterion for functional recovery [20]. However, the key issue always remained in finding out ways to integrate this strategy in neurorehabilitation [21]. Buch et al. conducted clinical trials on eight stroke patients with chronic hand plegia wherein they were given a BCI triggered orthotic feedback, but it yielded no significant functional recovery [22]. In another study RamosMurguialday et al. have found significantly higher motor recovery in case of BCI based contingent exoskeleton feedback compared to random exoskeleton feedback [23]. Ang et al. compared different rehabilitation strategies involving both MP and PP to prove that BCI based concomitant robotic feedback has more promising outcome rather than simply BCI triggered sensorimotor feedback [21]. Prasad et al. have also found that 


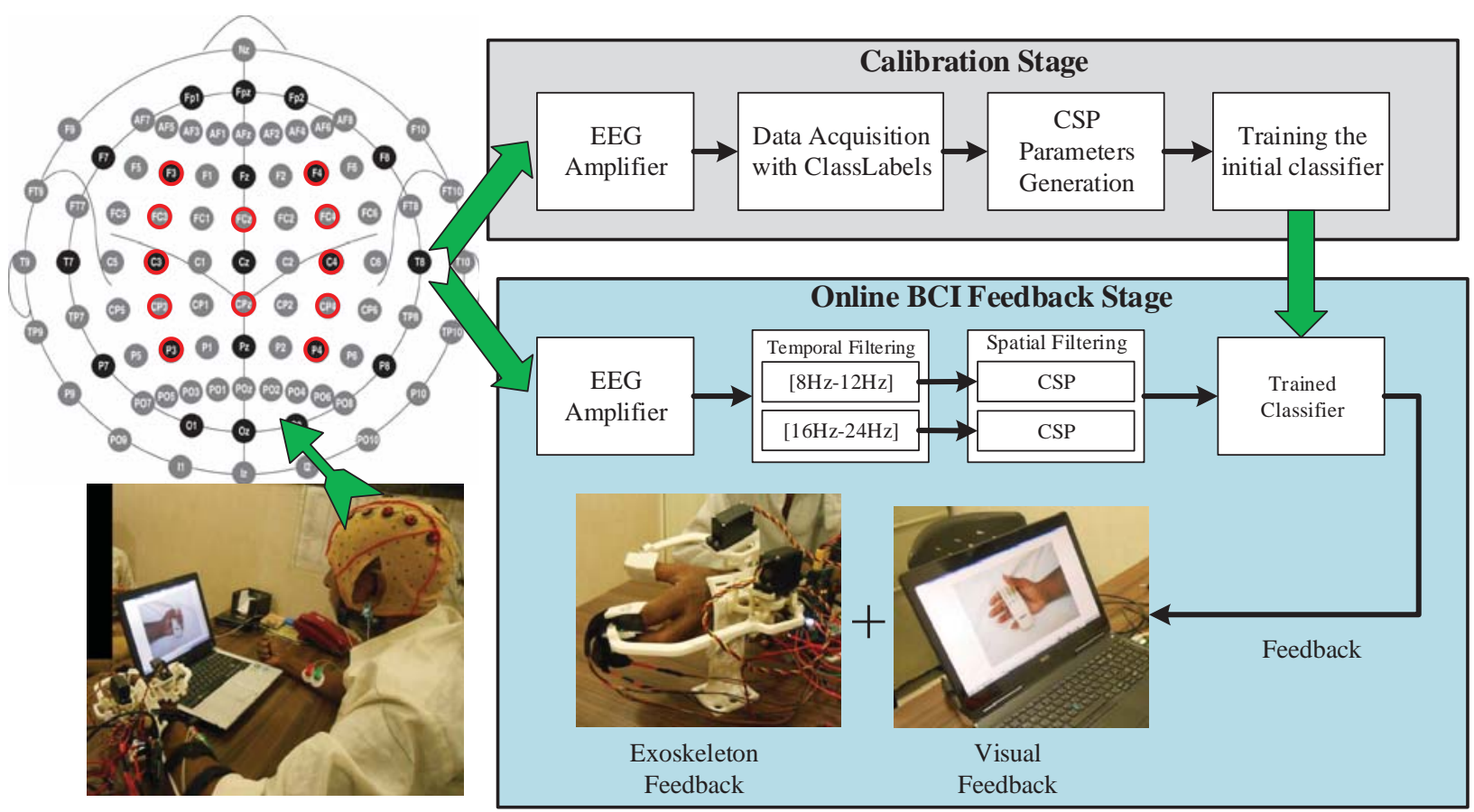

Fig. 1. An overview of the EEG-based BCI supported neurorehabilitation system. The selected EEG channel distribution is marked in red colour and shown in the upper left corner, and a participant undergoing the experimentation is shown in the bottom left corner. The top right and bottom right sections of the figure are showing the different components of the BCI calibration phase and the online feedback generation phase.

the combination of MP and PP is a feasible rehabilitation protocol, as significant improvement in ARAT measurement was observed between pre and post intervention [24].

The multimodal feedback comprising of different combinations of visual, auditory and proprioceptive means, happens to be more encouraging for the patients, leading to improved performance [25], [26]. Darvishi et al. also found it important to design an optimal feedback update interval (FUI) for better rehabilitative outcome during their clinical study on stroke patients [27]. The strategy of attempting a movement rather than only imagining has been suggested by many researchers as it helps subjects to focus more on the movement they are imagining which in-turn improves the BCI performance [28], [1].

Most of the aforementioned studies thus either dealt with PP only or they used BCI driven MP with or without the exoskeleton. As these studies reported some sort of motor recovery using either of these two techniques, researchers went on comparing them ([14]) or combining them ([24]) to test their effectiveness in various aspects. Another key issue apart from testing the clinical efficacy is the usability of these neurorehabilitation paradigms which are mostly not reported except in the case of Morone et al. [17] and Prasad et al. [24]. A large-scale clinical trial by Ang et al. [21] compared the effect of BCI triggered feedback to the concomitant feedback using exoskeletons although no separate PP stage was there in that study. The work of Prasad et al. [24] included separate PP, although the PP stage was manual, having no active robotic assistance and MP part had only BCI based visual feedback in terms of a computer game. The novelty of the current study lies not only in adding a separate hand-exoskeleton assisted active PP stage before the BCI driven hand-exoskeleton based MP stage, but in investigating both the clinical effectiveness and the usability of the system. Additionally, it provided both the anthropomorphic visual and proprioceptive neurofeedback, while most of the previous studies used either of these. Thus in terms of combining all the key elements of a neurorehabilitation protocol (which are distributed in various previous studies) and testing both the clinical effectiveness and usability, the current study has a novel contribution in line with the state-of-the-art neurorehabilitation research.

Industry 4.0 focuses on enhancing automation employing cyber-physical systems that can automate processes and monitor physical processes and take decisions using Internet-ofThings (IoT) and Artificial Intelligence (AI) over the internet. The current study has the inherent potential to align the field of neurorehabilitation with the industry 4.0 architecture. First it automates the manual rehabilitation therapy using sensor based observation of user's physical/mental engagement during the therapeutic process and helps the user carrying out exercise with a robotic device. Second, the system is designed as a graphical user interface (GUI) based operating interface with an MS-access database, so that it can facilitate remote monitoring, as well as evaluation of the rehabilitation progress while connected to the internet.

Clinical trials were conducted for 12 to 16 sessions (over 
the span of 6 weeks, i.e. $2-3$ sessions/week) on 4 hemiparetic stroke patients with partial disability in finger movements. ARAT and GS of the affected hand were measured to assess their functional recovery. The mood, fatigue, and motivation were also measured by a $10 \mathrm{~cm}$ visual analog scale (VAS) as part of usability studies. The results suggest that the proposed rehabilitation system has a great potential to be a clinically effective and usable solution for post-stroke hand function recovery.

\section{Methods And Materials}

\section{A. Participant recruitment}

The inclusion criteria for the patients were as follows. Male and female post-stroke volunteers, in the age group of 18-80 years, having movement disability in at least one of their hands due to stroke and having normal or corrected to normal vision (e.g. normal vision by using glasses) were considered for the study. The participants should be no less than 6 months poststroke since the first episode of stroke: This is to ensure the stage of fast spontaneous recovery has finished. They should be able to follow two-part spoken or written commands: This is to ensure stroke survivors can provide informed consent and also to ensure they will be able to comply with therapy. The patients were excluded from the study if they have a progressive neurological condition, any serious medical or psychological diseases which are likely to seriously affect their ability to continue with experimentation or they are known to suffer from epilepsy. The patients' gross cognitive impairment or disorientation were tested using Hodgekinson Mini-Mental State Examination (HMMSE), and those who scored $<7$ were excluded. The presence of moderate to high muscle spasticity and/or tremor in hand was also set as an exclusion criterion. No BCI screening was performed during the recruitment of the patients. We have confirmed from the testimonials of the patients that they stopped recovering after 2-3 months poststroke and they were not going through any kind of physiotherapy in parallel during the clinical trial period. The study was approved by the ethics committee of Indian Institute of Technology (IIT) Kanpur, India (IITK/IEC/2016-17/8) and the participants gave their written consent before the trials. The trial is registered with CTRI and is assigned a registration number: CTRI/2018/05/013876.

Patients were recruited from the Kanpur district of India. Their demographics are shown in Table I. As per the "CONSORT" flow-diagram the outline of the clinical trial is reported as follows. During the enrollment of the trial a total of 16 patients were assessed for eligibility out of which 11 patients were excluded for various reasons such as 10 of them didn't meet the inclusion criteria, and 1 of them declined to participate due to long travel time from home to the center. Although 5 patients were selected initially and received the allocated intervention, one of them discontinued intervention after 2 trials due to some personal reasons and hence excluded from analysis. Thus, the analysis was done for the rest of the 4 participants who completed a minimum of 12 therapeutic sessions as per the allocated intervention.

\section{B. Experimental protocol}

To ensure sufficient dose it has been suggested to go for six weeks of therapy with three sessions per week to expect any positive outcome [29]. In line with this recommendation, participants underwent a total of 12 to 16 therapy sessions spanned over 6 weeks with 2-3 sessions per week. In a session for the first $30 \mathrm{~min}$, the participants did PP with a hand exoskeleton attached to their affected hand. The exoskeleton was operated in an assist-as-needed mode to perform repetitive flexion and extension motion of their thumb, index and middle fingers. This was followed by $16 \mathrm{~min}$ of $\mathrm{BCI}$ calibration time before entering into the actual MP phase, which continued further for another $30 \mathrm{~min}$. The BCI calibration phase was composed of two runs of 40 trials each equally divided between left-hand and right-hand motor tasks. The objective during the MP phase is to perform left or right-hand finger extension attempts according to random cues provided on the computer screen.

Although a large section of the BCI systems for rehabilitative use are based on motor imagery without overt movement [30], patients have compromised-ability for brain-wave modulations related to the motor-task, leading to improper neurofeedback, which in turn induces frustration among the patients and further degradation of the performance [31]. As a motor imagery is often found to be less natural and hence difficult for the patient to perform, resulting in less distinguishable features [28], the participants were advised to attempt the movement. In particular, the patients were instructed to repetitively attempt the movement with a pace as slow as possible until a single trial is over. The experimental paradigm and the neurofeedback along with the timing diagram of the trials are depicted in Fig. 1 and Fig. 2 respectively. The BCI calibration needed approximately $16 \mathrm{~min}$ to complete. The MP was composed of 3 runs of BCI based neurofeedback, each consisting of 40 trials. There was 2.5 min of break period after each run of the feedback phase.

\section{System overview and data acquisition}

The current sources associated with the finger movement are found to be in the frontal medial and parietal regions of the brain as revealed by the joint fMRI and EEG studies [32]. The EEG channel description can be found in Fig. 1. The sampling rate for data acquisition was $512 \mathrm{~Hz}$. The signals were bandpass filtered between 0.1 to $100 \mathrm{~Hz}$ and notch filtered at 50 Hz. The study used the bio-signal amplifier g.USBamp (g.tec, Graz, Austria), along with active ring electrodes (g.LADYbird having sintered $\mathrm{Ag} / \mathrm{AgCl}$ crown) attached to the EEG cap (g.GAMMAcap). The reference electrode was linked to the left earlobe. An in-house GUI supported software developed in MATLAB/SIMULINK ${ }^{\mathrm{TM}}$ platform was used for processing the EEG signal and generating neurofeedback.

The hand exoskeleton [33] used to facilitate the exercise of the impaired hand was built in-house which provides flexion and extension motion to the thumb, index and middle fingers. The index and middle fingers are coupled together and are driven by a four-bar mechanism. The thumb is driven by another separate four-bar linkage. The mechanisms are 


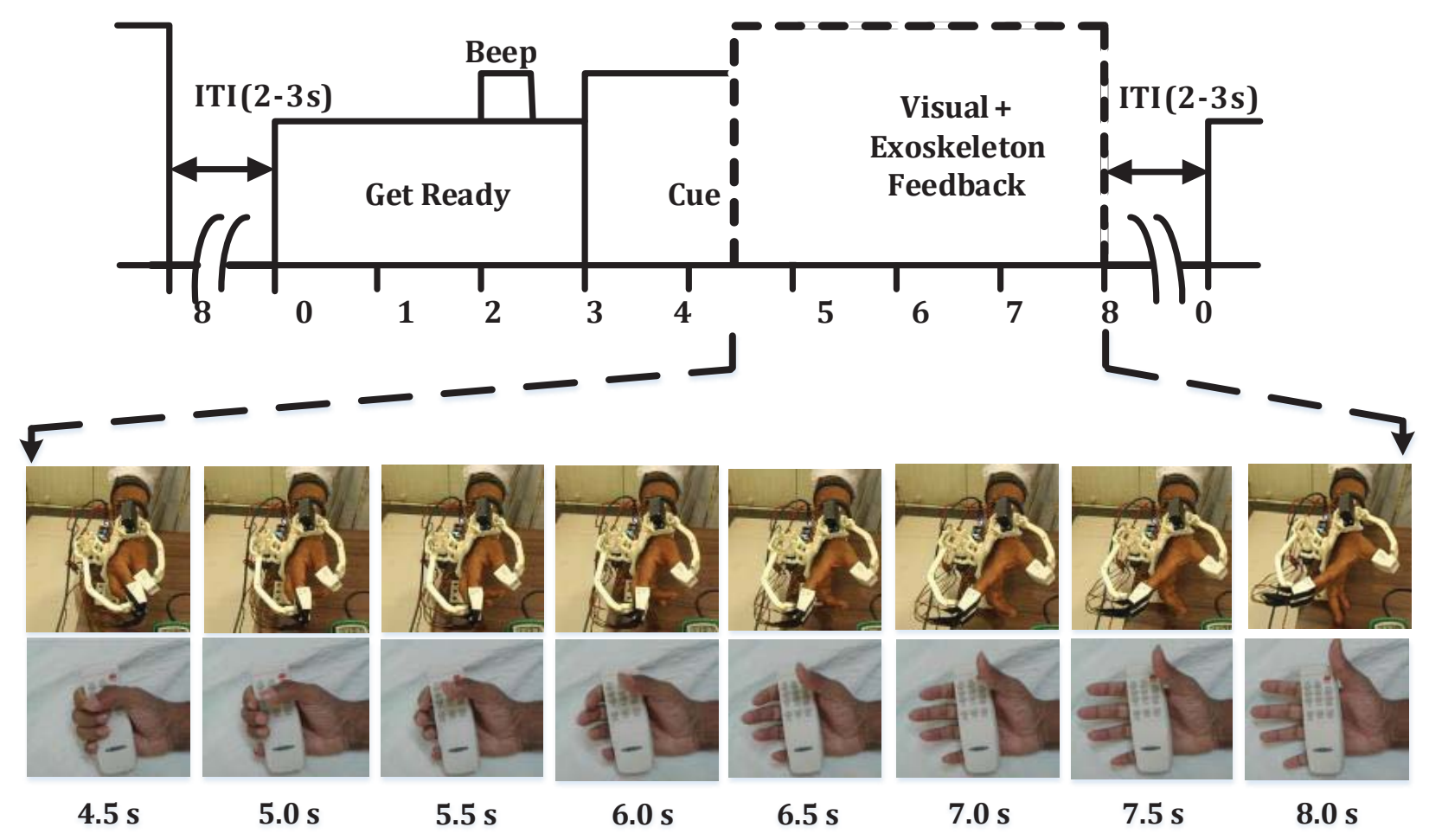

Fig. 2. The timing diagram and neurofeedback modalities. The feedback starts at $4.5 \mathrm{~s}$ and it has 8 instants of feedback generation at every $0.5 \mathrm{~s}$ up to $8 \mathrm{~s}$. Each instant with time stamp is represented below the timing diagram. The first row from the top is the sequences of exoskeleton actuation and the next row is showing the frame of the stop motion video. Both the feedback modalities go from fully closed at the beginning to fully opened position at the end if all the time points are classified correctly, otherwise the frame and the actuation sequence vary accordingly.

TABLE I

BASEline DEMOgRaphics OF THE PARTICIPATED PATIENTS

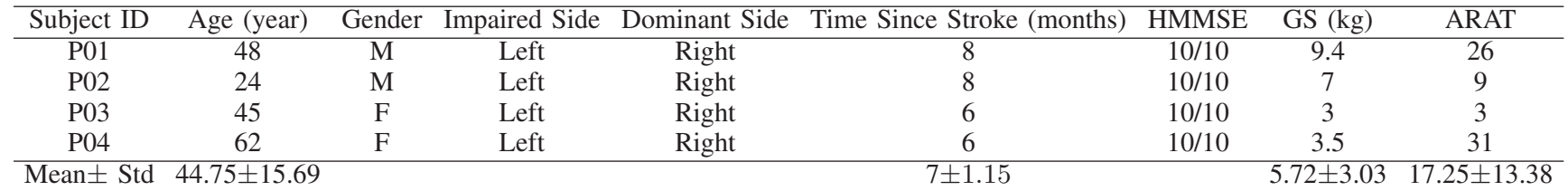

actuated by high torque servo motors in a position control mode. The exoskeleton was fabricated using a nylon based material to make it lightweight, flexible and portable. All the safety precautions were taken while using it on the stroke patients so that it was comfortable to wear and did not apply unnecessary force on the fingers. The entire system is portable and suitable for a quick installation in any place outside the lab environment, such as in a hospital.

\section{Physical practice}

The physical practice (PP) was carried out with the help of the hand exoskeleton attached to the patients' impaired hand and the unimpaired hand rested freely. Participants were instructed to perform 10 repetitions of the flexion and extension motion of their fingers alternatively using their impaired and unimpaired hands. For example, 10 flexion and extension first with the impaired hand, followed by 10 flexion and extension using unimpaired hand and so on. In order to encourage the patients in using their residual muscle strength, the exoskeleton was operated in assist-as-needed mode. This was done using force sensitive resistors (FSRs) attached on to the point of contact between the fingertips and finger caps, of the exoskeleton end-effector as shown in Fig. 3. The FSRs can sense the forces applied by the fingers in the flexion or extension direction and convert them into exoskeleton motion using an impedance control approach. Generally, patients don't have the full range of motion in their fingers and often fail to exert any force by their fingertips. In such situations, the exoskeleton controller simply moves the finger in the required direction at a predefined constant velocity. Apart from this, there are also the cases where a patient tends to exert the force in the opposite direction during an extension motion due to spasticity. To deal with such scenarios the exoskeleton controller keeps track of the movement phase (i.e. flexion or extension) and only considers the forces which are in 


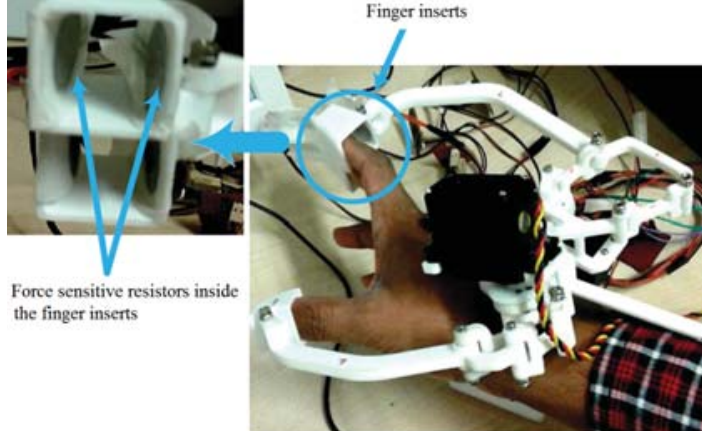

Fig. 3. The exoskeleton worn by the user is shown in the right side of the figure while the enlarged view of the finger insert showing the force sensitive resistors attached inside is shown in the left side of the figure.

accordance with the phase. The controller updates the phase information only after an ongoing motion (flexion/extension) is completed. This strategy prevents the patients to depend fully on external assistance which is essential for motor skill learning according to the guidance hypothesis [34].

\section{E. Multimodal neurofeedback during MP}

The multimodal neurofeedback comprises twin modes of visual and proprioceptive feedback, as discussed below:

1) Visual feedback: In order for a neurofeedback to be biologically relevant and intuitive, Folds et al. [35] have shown the utility of a stop motion video of a hand grasping action and demonstrated that the grasp aperture can be controlled by an MEG-based BCI system, leading to significant improvement in the SMR modulation of three spinal cord injury (SCI) patients. In the current study, we also have implemented a similar visual feedback mechanism consisting of a stop motion video to visualize the finger extension motion. The aperture of the finger was divided into 8 steps between 0 to $100 \%$. The $0 \%$ corresponds to the fully closed position and $100 \%$ corresponds to the fully open position; the rest of the steps are divided evenly. The EEG classifier generates the output at steps of $0.5 \mathrm{~s}$ from $4.5 \mathrm{~s}$ to $8 \mathrm{~s}$ of the trial duration. Upon every successful classification the aperture opens by 1 more step. Thus if it correctly classifies at all the 8 time points that means the finger aperture will open $100 \%$ and if only half of them are classified correctly then $50 \%$. As long as the classifier fails to detect the correct class, the video stays at the current frame. Thus the visual feedback is aimed at engaging the patient's focus throughout the trial period and encouraging him/her put more and more effort which is quintessential for motor skill learning. The gradual extension of the virtual finger during the neurofeedback period is shown frame by frame in Fig. 2.

2) Proprioceptive feedback: Proprioceptive feedback is related to feeling the movement of different body parts. It can thus carry the sensory information generated by the paretic limb movement and help recruit the motor areas around the lesion resulting in functional recovery and improved BCI performance [36]. Here, we have used the three-finger hand exoskeleton for giving proprioceptive feedback of the patients' thumb, index and middle finger extension motion. The movement of the exoskeleton is coupled with the visual feedback, which in turn depends on the classifier output as explained earlier. Fig. 2 shows the exoskeleton movement during a BCI trial alongside the visual feedback.

\section{F. Signal processing method}

1) Feature extraction: It is known that the ipsilateral enhancement of the lower $\beta$ rhythm and contra-lateral attenuation of the $\mu$ rhythm are normally related to motor execution as well as motor imagery [24] and are represented by neuro-physiological phenomena of event-related synchronization (ERS) and desynchronization (ERD). Therefore the temporal filtering of the EEG signals was done in two passbands of [8-12] $\mathrm{Hz}$ ( $\mu$ band) and [16-24] $\mathrm{Hz}$ ( $\beta$ band), as they were empirically found to generate relatively stable ERD/ERS patterns [37].

The spatial filters were computed using common spatial patterns (CSP) algorithm, which is used to maximize the discrimination between two classes [38]. The data covariance matrices from the two different classes are diagonalized simultaneously to define the spatial patterns [39]. The CSP algorithm carries out a supervised decomposition of signals which is parameterized by a matrix $W \in \mathbb{R}^{C \times C}$ (C: number of channels). This matrix is used to project the original sensor space $E \in \mathbb{R}^{c}$ into the surrogate sensor space $Z \in \mathbb{R}^{c}$. The spatial filter maximizes the difference in variance of the two classes of EEG signals. A small number of spatially filtered signals are used as features for classification purposes. Generally, $m$ first and $m$ last rows of $Z$ are selected, i.e. $Z_{t}$, where $t \in\{1 \ldots 2 m\}$. The feature vector $x_{t}$ is thus computed using the log-variance of $Z_{t}$.

2) Classifier design: The features were extracted from different time points over the trial length after the cue appearance. We have calculated CSP features at 8 time points from $4.5 \mathrm{~s}$ to $8 \mathrm{~s}$, along the trial length with a time step of $0.5 \mathrm{~s}$ (i.e. at $4.5 \mathrm{~s}, 5 \mathrm{~s}, 5.5 \mathrm{~s}, 6 \mathrm{~s}, 6.5 \mathrm{~s}, 7 \mathrm{~s}, 7.5 \mathrm{~s}$, and $8 \mathrm{~s}$ ). For each time point the data of the previous 1.5 s (i.e. 768 samples) were considered for CSP feature calculation. After performing the temporal and spatial filtering, and taking the log variance, we obtain a two-element feature vector for each frequency band $\mu$ and $\beta$. Thus from two different frequency bands (i.e. $\mu$ and $\beta$ ) we get four elements which form the feature vector of $1 \times 4$ dimension at a single time point. Similarly, the features were calculated from all the 80 trials (spanning two runs of 40 trials), divided into two classes for the BCI calibration phase. An support vector machine (SVM) classifier model was trained with these features using a linear kernel. Here we have trained 8 different classifier models for 8 time points. The calibration was participant specific and it was done in each session before the feedback phase. In the feedback phase, the classifier used these different classifier models to predict the left-hand and right-hand classes at each time point in a trial. On each successful prediction a counter, (initialized to 0 at the beginning of each trial) is incremented by 1 , and thus drives the contingent visual and exoskeleton feedback step by step.

\section{G. Rehabilitation outcome measures}

The rehabilitation outcomes were measured using GS and ARAT, as their incremental changes were found to have 


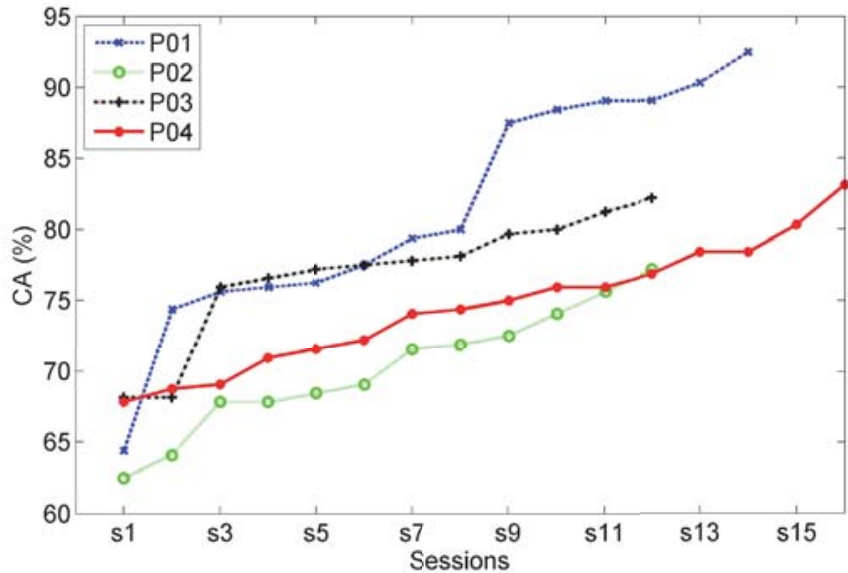

Fig. 4. The change in the classification accuracy (CA) through the therapy sessions.

significantly larger correlation with the variations in ERD/ERS for all the participants in our previous study [24]. Grip strength measurement using dynamometer (Camry ${ }^{\mathrm{TM}}$ Electronic Hand Dynamometer) is a standard technique for assessing the muscle strength of an individual after stroke. ARAT measurement, introduced by Lyle et al. [40] is also a reliable way of testing the upper-limb functionality by checking the grasp, grip, pinch and gross-movement activities. The apparatus used for the test are blocks of wood of different sizes, sharpening stone, cricket ball, glass and jar of water, hollow tubes of different height and thickness, washers, ball bearings, and marbles of different dimensions. The total score associated with the test is 57 which is distributed among different tasks with different apparatus listed above, where each task is scored between 0 and 3. All tests were administered under the supervision of an occupational therapist stationed at the hospital where the clinical trials were conducted.

\section{H. Usability measures}

The usability of the proposed rehabilitation system was analyzed in terms of the $10 \mathrm{~cm}$ visual analog scale (VAS) scores of fatigue, mood, and motivation of the participants during each therapy session. For the fatigue level, $0 \mathrm{~cm}$ in the VAS scale was marked as 'No fatigue', while $10 \mathrm{~cm}$ was marked as 'Worst fatigue imaginable'. For mood 'Lively mood' was marked at $0 \mathrm{~cm}$ while 'Worst mood' was marked at $10 \mathrm{~cm}$. In the motivation VAS scale 'Mastery confidence, I am looking forward for the task' was marked at $10 \mathrm{~cm}$ while 'Anxiety about failing the task' was marked at $0 \mathrm{~cm}$. The qualitative comments about the usability of the hand exoskeleton were also recorded using a standard questionnaire.

\section{RESULTS}

The results are presented to support the primary and secondary objectives of the study. To support the primary objective of the study, which is to test the possible clinical effectiveness of the proposed neurorehabilitation protocol, the essential measures are obviously the motor recovery outcomes
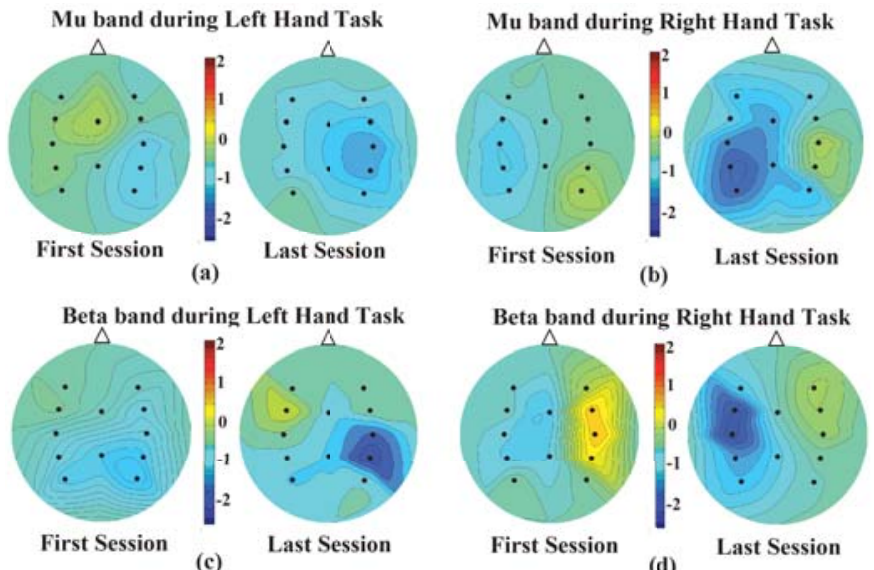

Fig. 5. The last vs. first session changes in scalp topoplots for (a) left-hand task in $\mathrm{Mu}$ band, (b) right-hand task in $\mathrm{Mu}$ band, (c) left-hand task in Beta band, (d) right-hand task in Beta band

such as GS and ARAT scores. However, it is also important to see the changes in the BCI performance and the neurophysiological effects in terms of scalp topoplots of EEG band powers during the therapy, so as to support the rehabilitative outcomes as this is a single arm study without a control group. Finding the relation between the individual BCI performance and the GS and ARAT measures could also be a verifier for a possible clinical effect. These results are presented in this section to support the primary objective. Moreover, the secondary objective, which is to test the usability of the system is reported by the VAS scale measurements of the mood, motivation, and fatigue.

\section{A. Results related to the primary outcome}

1) The BCI performance and the neurophysiological effect: The performance of the rehabilitation system was evaluated based on the BCI classification accuracy (CA). For calculating the $\mathrm{CA}$ for each session we averaged the CA calculated at all 8 time points along the trial length. The average CAs thus calculated for each participant for all the sessions are plotted in Fig. 4. The graph shows an increasing trend in accuracy for all the participants. We have calculated paired t-test (which is commonly used in pre and post observations on the same participants) to compare the accuracies of the last vs. first session across all the participants, which shows that the accuracy improvement is statistically significant at the $\mathrm{p}<0.05$ level $(p=0.0120)$. The CA averaged over all the sessions was found to be $81.45 \pm 8.12 \%, 70.21 \pm 4.43 \%$, $76.88 \pm 4.49 \%$, and $74.55 \pm 4.35 \%$ for $P 01, P 02, P 03$ and $P 04$, respectively. This shows that the performance of the BCI meets the recommended minimum accuracy level of $70 \%$ for controlling an external device [41]. A group mean change of +18.05 was also observed between the first and last session. The $\mu$ and $\beta$ band ERD/ERS topoplots are also shown in Fig. 5 to highlight the changes in the brain activity in first and last session. The ERD/ERS has been measured using (1).

$$
E R D / E R S_{b}^{c h}=\frac{E_{t a s k}^{b}}{E_{r e f}^{b}}
$$


where $b$ is the band ( $\mu$ or $\beta$ ) and $c h$ is the EEG channel. If the ratio ERD/ERS is less than 1 then ERD occurs and if it is greater than 1 then it is ERS. In the current study the reference time window was fixed between $2 \mathrm{~s}$ to $2.5 \mathrm{~s}$ within a trial. In order to discuss more specifically about the EEG channels and frequency bands, whose modulations changed in the course of the clinical trial, Fig. 5(a-d) has been depicted. Due to space limitations we have shown the topoplots based on the grand averages of ERD/ERS across all the participants, rather than showing the participant specific changes. The mu band scalp topoplot is shown in Fig. 5(a), which showed significantly $(\mathrm{p}<0.05)$ enhanced ERD (last vs. first) in C4 and CP4 channels in the ipsilesional primary and somatosensory motor cortex during left-hand (the impaired side) task. In harmony with the previous findings [42], significantly $(p<0.05)$ increased ipsilesional beta desynchronization (last vs. first) can also be located in the same $\mathrm{C} 4$ and $\mathrm{CP} 4$ channel locations from Fig. 5(c). The level of ERD in beta at $\mathrm{C} 4$ and CP4 were also found to be greater $(p<0.05)$ than the corresponding mu ERD indicating the relevance of the beta band in motor skill learning as reported in the previous studies [43]. During right-hand task the significantly $(\mathrm{p}<0.05)$ enhanced mu band ERD can be seen at the contralesional CP3 and P3 locations(see Fig. 5(b)), whereas significant $(p<0.05)$ beta ERD increment can be found in contralesional C3 and FC3(see Fig. 5(d)), when compared between the last vs. first session. The increased ERD also contributed to the segregation of the CSP features between left and right-hand tasks, which led to the enhancement in the classification accuracy.

2) Rehabilitation outcome measures: Rehabilitation outcome was measured using GS and ARAT scores on the impaired hand (i.e. left hand). Variations in GS and ARAT starting from the baseline at the commencement of the first session to the end session are shown in Fig. 6(a) and Fig. 6(b) respectively. As shown in Fig. 6(a), all the participants were able to increase their GS through the therapy sessions with a group mean change of $+6.38 \mathrm{~kg}$, although due to small sample size the paired t-test yielded a $p$-value $=0.06$, which was greater required level of $p$-value $<0.05$ for statistical significance. Substantial changes in the ARAT scores were also found for all the participants as shown in Fig. 6(b), with a statistically significant $(p$-value $<0.05)$ group mean change of +5.66 .

Pearson's correlation coefficients between CA vs. GS, and CA vs. ARAT are also shown in Fig. 6(c), to show the effect of BCI performance on rehabilitation outcomes. The correlation has been calculated by considering CA, GS and ARAT measures as a time-series of length equal to the number times the outcome measures were taken. As the GS and ARAT measurements are taken on each alternative sessions, the CA values corresponding to those sessions are considered while calculating the correlation. The correlation coefficients between CA and GS were 0.79, 0.92, 0.84, and 0.96 for $P 01$, $P 02, P 03$, and P04, respectively, while the correlation coefficients between CA and ARAT were 0.82, 0.98, 0.90, and 0.98, respectively. The correlations were statistically significant with $p$ value $<0.01$. Thus both the outcome measures were strongly correlated with the CA. It is to be noted that although the BCI performance and motor-outcome measures significantly correlated individually(intra-participant), the correlations were not significant while considering it across the participants(interparticipant), i.e. correlating first and last session differences of CA vs. GS and ARAT measures.

\section{B. Results related to the secondary outcome}

The variation of the fatigue, mood, and motivation VAS scores through the therapy sessions are shown in Fig. 7. The group mean changes for fatigue, mood, and motivation were observed to be $-0.98,-1.38$ and +2.10 . Although the group mean change of fatigue is indicating only a slight decrease, which may not be very significant but at least the fact that the intervention didn't increase the fatigue level is a positive aspect of the study as one of the previous clinical trials reported a moderate increase in fatigue level [24]. Moreover, reductions in the group mean of the mood VAS score and an increase in motivation VAS score were found during the trials, which means that both the mood and the motivation level of the participants improved.

\section{DISCUSSION}

\section{A. Comparison of the BCI performance}

As compared to an earlier study consisting of PP without exoskeleton and MP [24], wherein the average classification accuracy of the BCI was in the range $60-75 \%$, a much higher accuracy in the range $70-81 \%$ was obtained. Unlike, the study in [24], all the participants were able to increase their BCI performance as the therapy session progressed. Many factors may have contributed to this outcome including the use of intuitive and multimodal neurofeedback mechanisms, the use of spatial filtering and increased number of EEG channels etc., although the scope of such speculations are limited by the lack of control groups. Particularly, the anthropomorphic feedback strategies are generally more engaging and intuitive for the participants which put the mirror neuron system into action, leading to stronger SMR activations [44]. Earlier studies also indicated that the contingent visual and proprioceptive feedback maximizes the information about the correctness of the BCI control [36], which could help the user streamline his/her actions during the BCI task, leading to improved performance. It is to be noted that once the EEG classifier was built after the calibration stage no adaptation was done in the online feedback generation stage; however, the calibration was done for every session separately. Therefore, the improvement in the accuracy depended on the participants' ability to generate distinctive EEG patterns for the left and right-hand tasks. Indeed, we didn't force the patients to generate a predefined pattern as done in the fixed model based operant conditioning; rather they were asked to focus on the motor task as much as possible, and the rest of the job was taken care of by the CSP feature extraction and SVM based classification at the calibration stage for subject-specific modeling. It is worth mentioning that although several algorithms for classifying the brain signals are tried and tested by various research groups, as in the case of BCI competition IV datasets [45], CSP based 


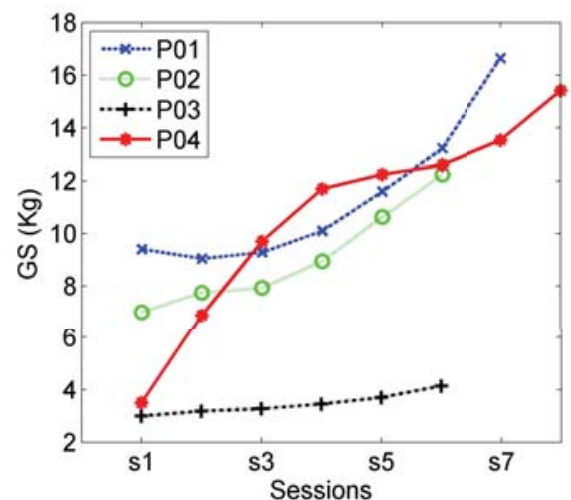

(a)

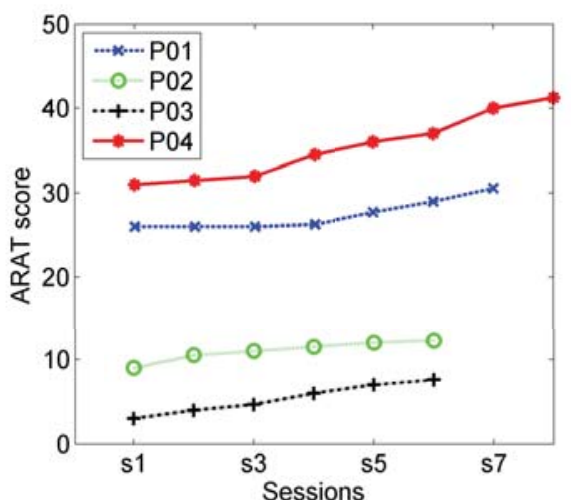

(b)

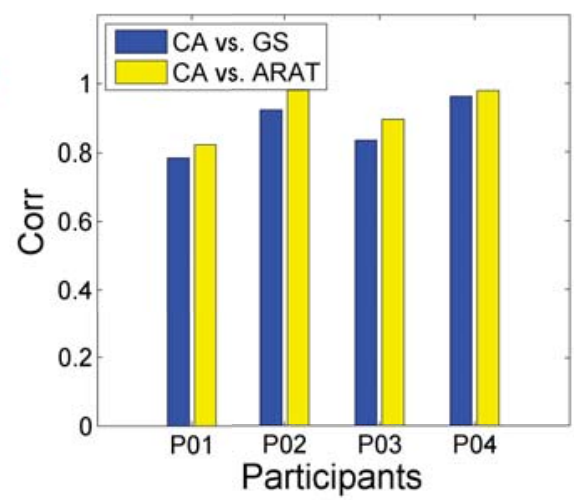

(c)

Fig. 6. Changes in the GS and ARAT through the therapy sessions and their correlation with the CA, (a) Change in the GS through the therapy sessions, (b) Change in the ARAT through the therapy sessions, (c) Correlation between (CA and GS), and (CA and ARAT).

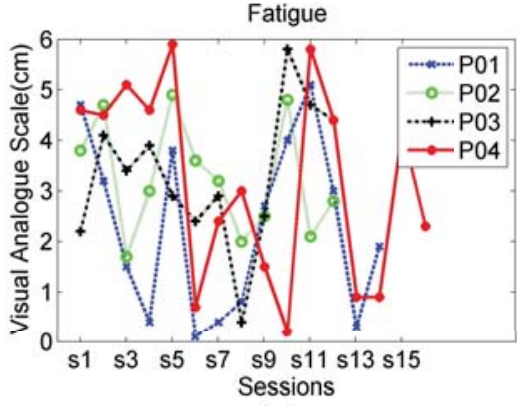

(a)

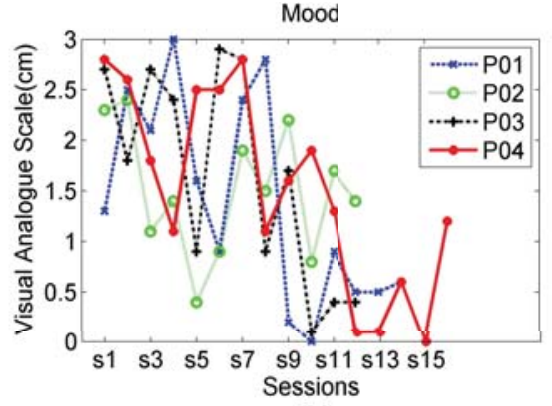

(b)

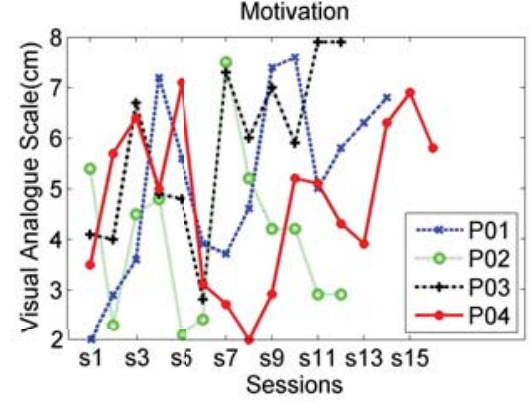

(c)

Fig. 7. VAS scores through the sessions, (a) fatigue, (b) mood, (c) motivation.

feature extraction is the most popular among the winners [46], [47]. In a recent study, Ang et al. [48] reported a clinical trial on 6 stroke patients with an average online feedback accuracy of $69.5 \%$. However, an adaptive strategy employed during offline analysis of data improved the accuracy by $12 \%$. The classification accuracies are also in keeping with a recently conducted large clinical study, where $60 \%$ of the participants achieved more than $70 \%$ accuracy [21].

\section{B. Comparison of the motor recovery measures}

As compared to the average baseline score of $5.73 \mathrm{~kg}$, participants were able to attain an increase $6.38 \mathrm{~kg}$ on an average which is a $111.49 \%$ improvement, while approximately $20 \%$ increment was reported in the earlier study [24]. Bundy et al. [49] also used a similar intervention (BCI based Hand exoskeleton), without the active PP part, where the average improvement in GS was found to be $22.64 \%$ (a change of $1.51 \mathrm{~kg}$, over a baseline of $6.67 \mathrm{~kg}$ ). In a recent study, the minimal clinically important difference (MCID) of GS was determined to be $19.5 \%$ of the initial value [50], which means the average improvement in GS in this case far exceeded the MCID limit. To ensure that increment in the GS is not due to increased spasticity, the spasticity level was regularly monitored by the occupational therapist. Moreover, the increment in ARAT scores requires both extension and flexion capabilities of a hand for grasping objects which also ensures that the spasticity level was not increased.

The average improvement in the ARAT score was 5.66 from the average baseline measurement of 17.25 , which is a $32.81 \%$ improvement. A study conducted by Darvishi et al. [27] achieved 36\% improvement in ARAT, whereas Bundy et al. [49] reported an average improvement in ARAT of $46.27 \%$ (i.e. increase of 6.2 over a baseline 13.4). In the current study, only one out of 4 participants achieved ARAT improvement beyond the MCID limit of 5.7, while in [24] and [49] it was 2 out of 5 and 6 out of 10 respectively. However, the percentage of average improvement in ARAT was comparable to the existing studies [24], [27], [49] and also very close to the MCID limit.

A few randomized controlled trials were also conducted for BCI based hand functional recovery, reporting better improvements compared to other rehabilitative paradigms. Ang et al. [51] conducted three-arm control trials to compare the effect of MI-BCI based haptic feedback with the only haptic feedback and conventional therapist assisted manual therapy and found significantly higher improvement in Fugl Meyer Assessment (FMA) in the MI-BCI based haptic feedback condition as compared to the other conditions. BCI based MI training was also proved effective as compared to the without BCI MI training in a controlled study by Pichiorri et al. [52] using anthropomorphic visual feedback of a virtual hand.

There is also a critical question often debated whether the 
BCI performance has any role to play in motor recovery. Some studies indicated that functional improvements in patients are associated with the classification accuracy of the BCI [53]. In the current clinical study also, we found a strong intraparticipant correlation between the BCI CA and the motor recovery measures GS and ARAT. Although [49] found significant inter-participant correlation between BCI performance and motor-outcome too, in our study this was not significant. There could be two possible explanations for this result: first is the smaller participant number (total 4 participants) as compared to [49] (total 10 participants); the second possibility could be that the influence of BCI performance on motoroutcome may suffer from inter-subject variability.

\section{Advantages and limitations}

The main advantage of the proposed neurorehabilitation protocol is that the manual PP strategy was replaced by a hand exoskeleton based assist-as-needed control, which enriches patients' engagement with the task. Also, the neurofeedback during BCI based MP has been improvised by the use of anthropomorphic visual and exoskeleton based proprioceptive feedback. Moreover, the human therapist can intervene in the rehabilitation process by setting different parameters in order to adjust the difficulty level of the physical and mental practice according to the recovery of the patients. This leads to more personalization of the therapeutic process, which is one of the major aspects of Industry 4.0 based healthcare. Thus the proposed neurorehabilitation system can be thought of as a prospective mode of telerehabilitation. The study also proposes a solution to the problem of shortage of expert human therapists needed for providing effective and personalized neurorehabilitation care, which is an emerging crisis for the aging world population.

The current study is limited by the small patient population, as to draw a statistical significance test for the recovery measures requires a larger group. Also, it would have been interesting to compare the effectiveness of the proposed MP+PP with exoskeleton paradigm, with other paradigms such as MP without PP or vice versa, or PP/MP without exoskeleton, which is not possible due to the lack of control groups. These issues will be catered in our future studies.

\section{CONCLUSION}

The pilot trial presented in this paper introduces a novel neurorehabilitation protocol incorporating a separate active hand exoskeleton based PP followed by BCI based MP with multimodal neurofeedback. The idea is to investigate the consequence of combining key neurorehabilitative features such as hand-exoskeleton based active PP, and contingent anthropomorphic visual and proprioceptive feedback. The improvement in BCI performance along with positive motorrecovery and mental state outcome measures show that the proposed neurorehabilitation protocol has a great potential to be a clinically effective and usable solution for hand functional recovery, although it needs further validation conducting controlled trials on the large patient cohort.

\section{ACKNOWLEDGMENT}

This work was supported in part by the Department of Science and Technology, India, and in part by the UKIndia Education and Research Initiative (UKIERI)-Thematic Partnership Project "A BCI Operated Hand Exoskeleton Based Neurorehabilitation System" under Grant UKIERI-DST-201314/126 and Grant DST/INT/UK/P-80/2014. The experimentation on patient groups were conducted in collaboration with the Regency Hospital, Kanpur, India.

\section{REFERENCES}

[1] U. Chaudhary, N. Birbaumer, and A. Ramos-Murguialday, "Braincomputer interfaces for communication and rehabilitation," Nature Reviews Neurology, vol. 12, pp. 513-525, 2016.

[2] S. R. Soekadar et al., "Brain-machine interfaces in neurorehabilitation of stroke," Neurobiology of Disease, vol. 83, pp. 172-179, 2015.

[3] B. H. Dobkin, "Rehabilitation after Stroke," New England Journal of Medicine, vol. 352, no. 16, pp. 1677-1684, 2005

[4] Z. Yue, X. Zhang, and J. Wangcorresponding, "Hand Rehabilitation Robotics on Poststroke Motor Recovery," Behavioural Neurology, vol. 2017, no. 3908135, p. 20, 2017.

[5] F. Shiman et al., "Classification of different reaching movements from the same limb using EEG," J Neural Eng., vol. 14, no. 4, p. 046018 , 2017.

[6] R. Bauer et al., "Bridging the gap between motor imagery and motor execution with a brainrobot interface," NeuroImage, vol. 108, pp. $319-$ 327, 2015.

[7] M. Vukelić and A. Gharabaghi, "Self-regulation of circumscribed brain activity modulates spatially selective and frequency specific connectivity of distributed resting state networks," Front Behav Neurosci., vol. 9, p. $181,2015$.

[8] D. Kraus et al., "Brain state-dependent transcranial magnetic closedloop stimulation controlled by sensorimotor desynchronization induces robust increase of corticospinal excitability," Brain Stimulation, vol. 9, no. 3, pp. 415 - 424, 2016.

[9] B. H. Dobkin, "Brain-computer interface technology as a tool to augment plasticity and outcomes for neurological rehabilitation," The Journal of Physiology, vol. 579, no. 3, pp. 637-642, 2007.

[10] T. Kawase et al., "A hybrid BMI-based exoskeleton for paresis: EMG control for assisting arm movements," J Neural Eng., vol. 14, no. 1, p. 016015, 2017.

[11] A. Chowdhury, H. Raza, Y. K. Meena, A. Dutta, and G. Prasad, "Online covariate shift detection based adaptive brain-computer interface to trigger hand exoskeleton feedback for neuro-rehabilitation," IEEE Transactions on Cognitive and Developmental Systems, pp. 1-1, 2017.

[12] G. Naros et al., "Reinforcement learning of self-regulated sensorimotor $\beta$-oscillations improves motor performance," NeuroImage, vol. 134, pp. $142-152,2016$.

[13] S. Darvishi et al., "Proprioceptive feedback facilitates motor imageryrelated operant learning of sensorimotor -band modulation," Frontiers in Neuroscience, vol. 11, p. 60, 2017.

[14] B. Várkuti et al., "Resting state changes in functional connectivity correlate with movement recovery for BCI and robot-assisted upperextremity training after stroke." Neurorehabilitation and neural repair. vol. 27, no. 1, pp. 53-62, 2013 .

[15] S. E. Fasoli et al., "Robotic therapy for chronic motor impairments after stroke: follow-up results," Archives of Physical Medicine and Rehabilitation, vol. 85, no. 7, pp. 1106-1111, 2004

[16] E. B. Brokaw et al., "Hand Spring Operated Movement Enhancer (HandSOME): A portable, passive hand Exoskeleton for stroke rehabilitation," IEEE Trans Neural Syst Rehabil Eng, vol. 19, no. 4, pp. 391-399, 2011.

[17] G. Morone et al., "Proof of Principle of a Brain-Computer Interface Approach to Support Poststroke Arm Rehabilitation in Hospitalized Patients: Design, Acceptability, and Usability," Archives of Physical Medicine and Rehabilitation, vol. 96, no. 3, pp. S71-S78, 2015.

[18] A. Chowdhury, H. Raza, A. Dutta, and G. Prasad, "EEG-EMG based hybrid Brain-Computer Interface for triggering hand exoskeleton for neuro-rehabilitation," in Proceedings of the Advances in Robotics, ser. AIR '17. New York, NY, USA: ACM, 2017, pp. 45:1-45:6.

[19] J. J. Daly et al., "Feasibility of a New Application of Noninvasive Brain Computer Interface (BCI): A Case Study of Training for Recovery of Volitional Motor Control After Stroke," Journal of Neurologic Physical Therapy, vol. 33, no. 4, pp. 203-211, 2009. 
[20] D. M. Nilsen, G. Gillen, and A. M. Gordon, "Use of mental practice to improve upper-limb recovery after stroke: A systematic review,' American Journal of Occupational Therapy, vol. 64, no. 5, pp. 695$708,2010$.

[21] K. K. Ang and C. Guan, "Brain-Computer Interface for Neurorehabilitation of Upper Limb After Stroke," Proceedings of the IEEE, vol. 103, no. 6, pp. 944-953, 2015.

[22] E. Buch et al., "Think to move: A neuromagnetic brain-computer interface (BCI) system for chronic stroke," Stroke, vol. 39, no. 3, pp. 910-917, 2008.

[23] A. Ramos-Murguialday et al., "BrainMachine Interface in Chronic Stroke Rehabilitation: A Controlled Study," Annals of Neurology, vol. 74, no. 1, pp. 100-108, 2013.

[24] G. Prasad et al., "Applying a brain-computer interface to support motor imagery practice in people with stroke for upper limb recovery: a feasibility study." Journal of neuroengineering and rehabilitation, vol. 7 , no. 1 , p. 60,2010

[25] T. Sollfrank et al., "The effect of multimodal and enriched feedback on SMR-BCI performance," Clinical Neurophysiology, vol. 127, no. 1, pp. 490-498, 2016

[26] M. Mihara et al., "Near-infrared spectroscopy-mediated neurofeedback enhances efficacy of motor imagery-based training in poststroke victims: A pilot study," Stroke, vol. 44, no. 4, pp. 1091-1098, 2013.

[27] S. Darvishi et al., "Investigating the impact of feedback update interval on the efficacy of restorative brain-computer interfaces," Royal Society Open Science, vol. 4, no. 8, p. 170660, 2017.

[28] Y. Blokland, L. Spyrou, D. Thijssen, T. Eijsvogels, W. Colier, M. FloorWesterdijk, R. Vlek, J. Bruhn, and J. Farquhar, "Combined EEG-fNIRS decoding of motor attempt and imagery for brain switch control: An offline study in patients with tetraplegia," IEEE Trans Neural Syst Rehabil Eng, vol. 22, no. 2, pp. 222-229, 2014.

[29] K. K. Ang et al., "Clinical study of neurorehabilitation in stroke using EEG based motor imagery brain-computer interface with robotic feedback," Proceedings of the 32nd Annual International Conference of the IEEE Engineering in Medicine and Biology Society, vol. 2010, pp. 5549-5552, 2010.

[30] A. Gharabaghi, "What turns assistive into restorative brain-machine interfaces?” Frontiers in Neuroscience, vol. 10, p. 456, 2016. [Online]. Available: https://www.frontiersin.org/article/10.3389/fnins.2016.00456

[31] R. Bauer and A. Gharabaghi, "Constraints and adaptation of closed-loop neuroprosthetics for functional restoration," Frontiers in Neuroscience, vol. 11, p. 111, 2017. [Online]. Available: https://www.frontiersin.org/article/10.3389/fnins.2017.00111

[32] T. Ball et al., "The role of higher-order motor areas in voluntary movement as revealed by high-resolution EEG and fMRI." NeuroImage, vol. 10, no. 6, pp. 682-94, 1999.

[33] Y. K. Meena, A. Chowdhury, H. Cecotti, K. Wong-Lin, S. S. Nishad, A. Dutta, and G. Prasad, "Emohex: An eye tracker based mobility and hand exoskeleton device for assisting disabled people," in 2016 IEEE International Conference on Systems, Man, and Cybernetics (SMC), Oct 2016, pp. $002122-002127$.

[34] C. Obayashi, T. Tamei, and T. Shibata, "Assist-as-needed robotic trainer based on reinforcement learning and its application to dart-throwing," Neural Networks, vol. 53, pp. 52-60, 2014.

[35] S. T. Foldes, D. J. Weber, and J. L. Collinger, "MEG-based neurofeedback for hand rehabilitation." Journal of neuroengineering and rehabilitation, vol. 12 , no. 1 , p. 85, 2015.

[36] A. Ramos-Murguialday et al., "Proprioceptive Feedback and Brain Computer Interface (BCI) Based Neuroprostheses," PLoS ONE, vol. 7 , no. 10,2012

[37] D. Rathee, H. Raza, G. Prasad, and H. Cecotti, "Current source density estimation enhances the performance of motor-imagery-related Brain-Computer Interface," IEEE Transactions on Neural Systems and Rehabilitation Engineering, vol. 25, no. 12, pp. 2461-2471, Dec 2017.

[38] B. Blankertz et al., "Optimizing spatial filters for robust EEG single-trial analysis," IEEE Signal Processing Magazine, vol. 25, no. 1, pp. 41-56, 2008.

[39] C. Park et al., "Augmented complex common spatial patterns for classification of noncircular eeg from motor imagery tasks," IEEE Trans Neural Syst Rehabil Eng, vol. 22, no. 1, pp. 1-10, 2014.

[40] R. C. Lyle, "A performance test for assessment of upper limb function in physical rehabilitation treatment and research," International Journal of Rehabilitation Research, vol. 4, no. 4, pp. 483-492, 1981.

[41] C. Vidaurre and B. Blankertz, "Towards a cure for BCI illiteracy," Brain Topography, vol. 23, no. 2, pp. 194-198, 2010.
[42] P. Belardinelli et al., "Plasticity of premotor cortico-muscular coherence in severely impaired stroke patients with hand paralysis," NeuroImage: Clinical, vol. 14, pp. 726 - 733, 2017.

[43] F. Khademi, V. Royter, and A. Gharabaghi, "Distinct beta-band oscillatory circuits underlie corticospinal gain modulation," Cerebral Cortex, vol. 28, no. 4, pp. 1502-1515, 2018.

[44] G. Buccino, A. Solodkin, and S. L. Small, "Functions of the mirror neuron system: implications for neurorehabilitation." Cognitive and Behavioral Neurology, vol. 19, no. 1, pp. 55-63, 2006.

[45] M. Tangermann et al., "Review of the BCI competition IV," Frontiers in Neuroscience, vol. 6, p. 55, 2012.

[46] H. Zhang et al., "BCI competition IV-data set I: Learning discriminative patterns for self-paced eeg-based motor imagery detection," Frontiers in Neuroscience, vol. 6, p. 7, 2012.

[47] K. K. Ang et al., "Filter Bank Common Spatial Pattern Algorithm on BCI Competition IV Datasets 2a and 2b," Frontiers in neuroscience, vol. 6, p. 39, jan 2012.

[48] K. K. Ang and C. Guan, "EEG-based strategies to detect motor imagery for control and rehabilitation," IEEE Trans Neural Syst Rehabil Eng, vol. 25, no. 4, pp. 392-401, April 2017.

[49] D. T. Bundy et al., "Contralesional Brain-Computer Interface control of a powered exoskeleton for motor recovery in chronic stroke survivors," Stroke, 2017.

[50] J. K. Kim, M. G. Park, and S. J. Shin, "What is the minimum clinically important difference in grip strength?" Clinical Orthopaedics and Related Research, vol. 472, no. 8, pp. 2536-2541, 2014.

[51] K. K. Ang et al., "Brain-computer interface-based robotic end effector system for wrist and hand rehabilitation: results of a three-armed randomized controlled trial for chronic stroke," Frontiers in Neuroengineering, vol. 7, p. 30, 2014

[52] F. Pichiorri et al., "Braincomputer interface boosts motor imagery practice during stroke recovery," Ann Neurol., vol. 77, pp. 851-865, 2015.

[53] S. Ruiz et al., "Acquired self-control of insula cortex modulates emotion recognition and brain network connectivity in schizophrenia," Human Brain Mapping, vol. 34, no. 1, pp. 200-212, 2013. 